\title{
Produtividade do sorgo forrageiro em função de doses de nitrogênio e manejo de
}

\section{cortes}

\author{
Forage sorghum yield as a function of nitrogen rates and cut management \\ Rendimiento de sorgo forrajero en función de las tasas de nitrógeno y el manejo del corte
}

Recebido: 13/08/2021 | Revisado: 22/08/2021 | Aceito: 23/08/2021 | Publicado: 25/08/2021

\author{
Gustavo de Faria Theodoro \\ ORCID: https://orcid.org/0000-0002-1230-2124 \\ Universidade Federal de Mato Grosso do Sul, Brasil \\ E-mail: gustavo.theodoro@ufms.br \\ Maryana Martins Ribeiro \\ ORCID: https://orcid.org/0000-0002-5112-8616 \\ Universidade Federal de Mato Grosso do Sul, Brasil \\ E-mail: maryana.ribeiro@outlook.com \\ Flávia Barbosa de Santana Pacheco \\ ORCID: https://orcid.org/0000-0002-6219-3034 \\ Universidade Federal de Mato Grosso do Sul, Brasil \\ E-mail: flavia.barbosa @ufms.br \\ Andrey William Alce Miyake \\ ORCID: https://orcid.org/0000-0001-7456-9513 \\ Universidade Federal de Mato Grosso do Sul, Brasil \\ E-mail: andrey.miyake@ufms.br
}

\begin{abstract}
Resumo
O sorgo forrageiro é uma planta com elevado potencial para o Centro Oeste brasileiro, especialmente por ser considerada tolerante a condições adversas e da elevada capacidade de perfilhamento e rebrote das soqueiras após ser cortado para silagem. $\mathrm{O}$ objetivo deste trabalho foi avaliar a influência de doses de nitrogênio $(\mathrm{N})$ e o manejo de cortes na produtividade do híbrido de sorgo forrageiro Agri 002E, cultivado na região central de Mato Grosso do Sul. O delineamento foi de blocos ao acaso, com quatro repetições, em esquema fatorial $2 \times 4$ e os fatores foram: duas épocas de corte e quatro doses de nitrogênio em cobertura $\left(0,50,150\right.$ e $\left.200 \mathrm{~kg} \cdot \mathrm{ha}^{-1}\right)$. As épocas de primeiro corte (PC) foram aos 63 (PC 63) e 97 (PC 97) dias após a semeadura. O experimento foi repetido após o PC até um segundo corte (SC), que ocorreu após 160 dias da semeadura. Foi avaliada a produtividade das plantas logo após cada corte. Considerou-se como manejo de corte ambos os cortes: 1. PC63, sucedido pelo SC 97 (PC63+SC97) e 2. PC97, sucedido pelo SC 63 (PC97+SC63). Independentemente do período de permanência das plantas ao campo, o sorgo produziu mais no primeiro corte e o efeito das doses de $\mathrm{N}$ na produtividade foi restrito ao período de 97 dias que precedeu o SC. O teor de matéria seca variou de 34,7 a 38,5\%. O PC97+SC63 foi mais eficiente por proporcionar as maiores produtividades de massa verde $\left(96,6\right.$ t.ha $\left.^{-1}\right)$ e seca $\left(37,9\right.$ t.ha $\left.{ }^{-1}\right)$.
\end{abstract}

Palavras-chave: Adubação; Sorghum bicolor; Sustentabilidade.

\begin{abstract}
Forage sorghum is a plant with high potential for the Brazilian Midwest, especially because it is considered tolerant to adverse conditions and the high capacity of tillering and regrowth of the ratoons after being cut for silage. The objective of this work was to evaluate the influence of nitrogen $(\mathrm{N})$ doses and cutting management on yield of the forage sorghum hybrid Agri 002E, cultivated in the central region of Mato Grosso do Sul. The design was a randomized block design, with four replications, in a scheme factorial $2 \times 4$ and the factors were: two cutting times and four doses of $\mathrm{N}$ in topdressing $\left(0,50,150\right.$ and $200 \mathrm{~kg}$.ha $\left.{ }^{-1}\right)$. The times of first cutting (FC) were 63 (FC63) and 97 (FC97) days after sowing (DAS). The experiment was repeated after FC until a second cut (SC), at 160 DAS. Plant yeld was evaluated right after each cut. Both cuts were considered as cutting management: 1. FC63, followed by SC 97 (FC63+SC97) and 2. FC97, followed by SC 63 (FC97+SC63). Regardless of the period of permanence of the plants in the field, sorghum yeld was higher in the first cut and the effect of $\mathrm{N}$ doses on yield was restricted to the 97-day period preceding SC. The dry matter content ranged from 34.7 to $38.5 \%$. FC97+SC63 was more efficient as it provided the highest yields of fresh $\left(96.6\right.$ t.ha $\left.^{-1}\right)$ and dry $\left(37.9\right.$ t.ha $\left.^{-1}\right)$ matter.
\end{abstract}

Keywords: Fertilization; Sorghum bicolor; Sustainability.

\section{Resumen}

El sorgo forrajero es una planta con alto potencial para el Medio Oeste brasileño, especialmente porque se considera tolerante a condiciones adversas y la alta capacidad de macollamiento y rebrote de los retoños luego de ser cortados para la ensilaje. El objetivo de este trabajo fue evaluar la influencia de las dosis de nitrógeno ( $\mathrm{N}$ ) y el manejo de la corta en el productividad del híbrido de sorgo forrajero Agri 002E, cultivado en la región central de Mato Grosso do Sul. El diseño fue un diseño de bloques 
Research, Society and Development, v. 10, n. 11, e109101119401, 2021

(CC BY 4.0) | ISSN 2525-3409 | DOI: http://dx.doi.org/10.33448/rsd-v10i11.19401

al azar, con cuatro repeticiones, en un esquema factorial $2 \mathrm{x} 4 \mathrm{y}$ los factores fueron: dos tiempos de corte y cuatro dosis de nitrógeno en topdressing $\left(0,50,150\right.$ y $\left.200 \mathrm{~kg} \cdot \mathrm{ha}^{-1}\right)$. Los tiempos de primer corte (PC) fueron 63 (PC63) y 97 (PC97) días después de la siembra (DS). El experimento se repitió después de PC hasta un segundo corte (SC), que se produjo 160 días DS. La productividad de la planta se evaluó inmediatamente después de cada corte. Ambos cortes fueron considerados como gestión de corte: 1. PC63, seguido de SC 97 (PC63 + SC97) y 2. PC97, seguido de SC 63 (PC97 + SC63). Independientemente del período de permanencia de las plantas en el campo, el sorgo produjo más en el primer corte y el efecto de las dosis de $\mathrm{N}$ sobre el rendimiento se limitó al período de 97 días anterior a la SC. El contenido de materia seca osciló entre el 34,7 y el $38,5 \%$. PC97+SC63 fue más eficiente ya que proporcionó los mayores rendimientos de masa verde $\left(96,6\right.$ t.ha $\left.{ }^{-1}\right)$ y seca $(37,9$ t.ha-1).

Palabras clave: Fertilización; Sorghum bicolor; Sustentabilidad.

\section{Introdução}

O sorgo (Sorghum bicolor L.) é uma espécie de relevante importância econômica para o Brasil, que cultivou 842,4 mil hectares e produziu 2,76 milhões de toneladas de grãos na safra 2020/21. Em Mato Grosso do Sul, constatou-se o maior aumento percentual de área cultivada com sorgo em relação à última safra (2019/20), pela valorização do preço do grão e à maior rusticidade da cultura (Conab, 2021).

Além de seu cultivo visando à produção de grãos, híbridos de sorgo podem ser destinados especificamente para a alimentação animal na forma de pastejo (Fernandes et al., 2020; Viana et al., 2020) ou silagem (Tiritan et al., 2013). No caso do sorgo forrageiro, a planta inteira é cortada para a produção de uma silagem com alta qualidade nutricional (Buso et al., 2011; Tolentino et al., 2016) e menor custo de produção (Costa et al., 2015; Rezende et al., 2020).

O sorgo é considerado uma espécie rústica e capaz de se adaptar a condições de baixa disponibilidade de nitrogênio (N) (Gardner et al., 1994). Entretanto, plantas nestas condições apresentam relevantes alterações estruturais, fisiológicas, morfogênicas e na absorção de outros nutrientes que comprometem a produtividade caso não haja a oferta de $\mathrm{N}$ por meio da adubação (Zhao et al., 2005; Silva et al., 2012; Santos et al., 2015).

Os relatos aparentemente discordantes em relação aos efeitos da adubação nitrogenada na produtividade da cultura do sorgo provavelmente estão relacionados à reação dos diferentes híbridos disponíveis (Silva et al., 2012) e sua aptidão de uso. Há relatos de incrementos na produtividade de grãos quando se empregaram doses de 120 kg N.ha ${ }^{-1}$ (Ramadhan et al., 2021) e $150 \mathrm{~kg} \mathrm{~N} \cdot \mathrm{ha}^{-1}$ (Abunyewa et al., 2017). Restelatto et al. (2013) verificaram que, dependendo do ano agrícola, as plantas de sorgo para pastejo produziram mais massa seca quando adubadas com as doses de $264\left(5,6\right.$ t.ha-1) e 288 kg.ha-1 $\left(12,9\right.$ t.ha $\left.{ }^{-1}\right)$. A ausência de efeitos na produtividade pelo uso de doses crescentes de $\mathrm{N}$ foi observada em experimentos com híbridos de sorgo sacarino (Scordia et al., 2021) e forrageiro (Oliveira et al., 2005; Rodrigues Filho et al., 2006; Marsalis et al., 2010).

Entre os híbridos de sorgo forrageiro existentes no mercado, encontram-se os que tem seu desenvolvimento limitado pela emissão da panícula com grãos viáveis e aqueles conhecidos como "gigantes", com um vigoroso crescimento vegetativo, elevada capacidade de perfilhamento, rebrota, ciclo maior e, com isso, a emissão tardia de uma panícula com pouco ou nenhum grão (Paziani et al., 2020).

As plantas podem permanecer por um longo período no campo em estádio vegetativo, com a possibilidade de ser realizado mais de um corte em função da elevada capacidade de rebrote (Botelho et al., 2010). Entretanto, o manejo de cortes precisa ser melhor compreendido em diferentes regiões produtoras, uma vez que aspectos edafoclimáticos interferem na morfofisiologia e no desempenho das plantas originadas do rebrote para uso na alimentação animal (Neumann et al., 2010; Schneider et al., 2019; Silva et al., 2020).

O objetivo deste trabalho foi avaliar se doses de nitrogênio e o manejo de cortes influenciam na produtividade de sorgo forrageiro cultivado na região central de Mato Grosso do Sul. 


\section{Metodologia}

O experimento foi conduzido na Fazenda Escola da Universidade Federal de Mato Grosso do Sul (UFMS), no município de Terenos, MS, nas coordenadas $20^{\circ} 26^{\prime} 17^{\prime \prime} \mathrm{S}$ e $54^{\circ} 51^{\prime} 24^{\prime \prime} \mathrm{W}$, a $407 \mathrm{~m}$ de altitude e com clima tropical e estação seca definida tipo Aw (KOTTEK et al., 2006). Os dados climáticos referentes ao local e período de condução do experimento foram apresentados na Figura 1 (CPTEC/INPE, 2021).

O solo foi identificado como Latossolo Vermelho distrófico (Embrapa, 2013), de textura muito argilosa, com as seguintes características químicas $(0-20 \mathrm{~cm}): \mathrm{pH}: 6,1 ; \mathrm{M} . \mathrm{O} .\left(\mathrm{g} \cdot \mathrm{kg}^{-1}\right): 32,4 ; \mathrm{Ca}\left(\mathrm{cmol}_{\mathrm{c}} \cdot \mathrm{dm}^{-3}\right): 5,0 ; \mathrm{Mg}\left(\mathrm{cmol}_{\mathrm{c}} \cdot \mathrm{dm}^{-3}\right): 5,8 ; \mathrm{K}$ $\left(\mathrm{cmol}_{\mathrm{c}} \cdot \mathrm{dm}^{-3}\right):$ 0,18; P $\left(\mathrm{mg}_{\mathrm{dm}}{ }^{-3}\right): 4,9, \mathrm{H}+\mathrm{Al}\left(\mathrm{cmol}_{\mathrm{c}} \cdot \mathrm{dm}^{-3}\right): 6,3$. Fez-se o preparo mínimo da área, conforme descrito por Theodoro et al. (2018) e a adubação na semeadura foi com $270 \mathrm{~kg} \cdot \mathrm{ha}^{-1}$ do formulado 10-10-10.

A semeadura ocorreu no dia 27/11/20118, empregando-se o híbrido de sorgo forrageiro Agri 002E, cultivado em espaçamento de 0,6 m entre linhas e uma população de 90.000 plantas por hectare. O híbrido Agri 002E, conhecido pelos produtores rurais como "sogo gigante boliviano", foi escolhido em função de sua alta capacidade de rebrote e perfilhamento (Latinaseeds, 2021), além de ser indicada como complementação volumosa em silagens (Paziani et al., 2020).

Figura 1 - Dados meteorológicos durante o período de cultivo do sorgo forrageiro no município de Terenos, MS. (CPTEC/INPE, 2021)

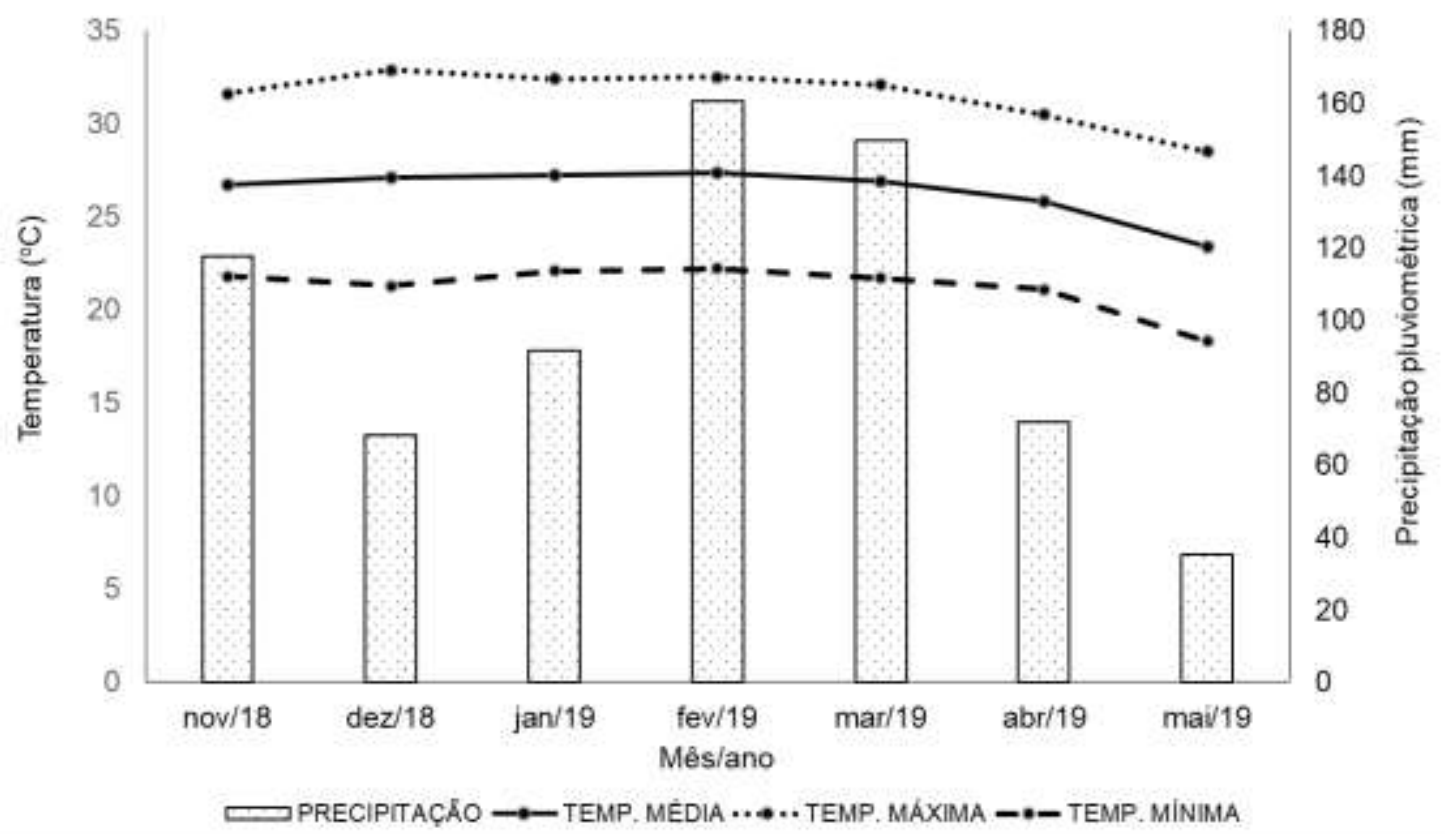

Fonte: Autores.

\section{Primeiro corte $(P C)$}

$\mathrm{O}$ experimento foi conduzido em delineamento de blocos ao acaso, com quatro repetições, em esquema fatorial $2 \times 4$. Os fatores foram: duas épocas de corte, aos 63 (PC 63) e 97 (PC 97) dias após a semeadura, que ocorreram respectivamente em 29/01/2019 e 04/03/2019 com o uso de uma roçadeira motorizada costal a 0,15 m do nível do solo (NS); e quatro doses de nitrogênio em cobertura: 0, 50, 150 e $200 \mathrm{~kg} \cdot \mathrm{ha}^{-1}$. A parcela experimental possuiu seis linhas de sorgo forrageiro, com cinco metros de comprimento, sendo considerada como área útil as duas fileiras centrais para que fosse evitado e efeito de bordadura. 


\section{Segundo corte $(S C)$}

O experimento foi realizado novamente, porém, efetuando-se um SC (0,15 m NS) nas plantas que surgiram após o rebrote das soqueiras remanescentes do PC. O delineamento experimental foi semelhante ao anterior, com as seguintes modificações: o sorgo do rebrote da soqueira das plantas do PC 63 recebeu um SC após 97 dias (SC 97); após o PC 97, procedeu-se a um segundo depois de 63 dias (SC 63).

\section{Manejos de corte (MC)}

Considerou-se como manejo de corte os dois que foram realizados durante o ciclo da cultura na área experimental, considerando-se os dados acumulados neste período. Desta forma, os tratamentos foram: 1. PC 63, sucedido pelo SC 97 (PC63+SC 97) e 2. PC 97, sucedido pelo SC 63 (PC97+SC63). Em ambos os tratamentos, a cultura permaneceu 160 dias no campo, momento em que foi realizado o segundo corte (06/05/2019). Após o PC, as plantas foram novamente adubadas em cobertura com as doses de $\mathrm{N}$ avaliadas, no mesmo estádio fenológico dos primeiros rebrotes.

\section{Tratos culturais}

A adubação em cobertura foi realizada com sulfato de amônio, quando as plantas estavam com cinco folhas expandidas após a semeadura e o rebrote da soqueira. Houve capina manual até os 30 dias após a semeadura e não foram aplicados agrotóxicos nas plantas durante o experimento. A incidência de pragas e doenças foi anotada durante o desenvolvimento da cultura.

\section{Avaliações}

As avaliações foram feitas em 20 plantas escolhidas aleatoriamente na área útil de cada parcela experimental. A primeira consistiu na mensuração da altura das plantas antes de cada corte, considerando-se o comprimento do colmo central do nível do solo até o último nó com inserção de folha. Imediatamente após os cortes, estimou-se a massa verde, massa seca e a matéria seca, empregando-se a metodologia descrita por Oliveira et al. (2005).

\section{Análise estatística}

$\mathrm{Na}$ análise dos resultados, consideraram-se os períodos compreendidos da semeadura até o PC; do rebrote das plantas até o SC; comparou-se o desempenho do PC com o SC, tanto aos 63 quanto aos 97 dias; e os dados acumulados em todo o período em que a cultura permaneceu no campo recebendo os MC.

Os dados foram submetidos a análise de variância e, quando pertinente, as médias comparadas pelo teste de Tukey a 5\% de probabilidade. Procedeu-se às análises de regressão de primeiro e segundo grau, considerando-se os efeitos por comparação (Perecin \& Cargnelutti Filho, 2008) e níveis de significância de 5\% de probabilidade, com a escolha do modelo de maior coeficiente de determinação.

\section{Resultados e Discussão}

\section{Primeiro corte}

Não houve interação significativa $(\mathrm{P}>0,05)$ entre doses de $\mathrm{N}$ e a época em que foi realizado o PC (Tabela 1). A adubação nitrogenada em cobertura não influenciou na produtividade da cultura no PC, porém, as plantas de sorgo apresentaram maior altura $(2,8 \mathrm{~m})$, massa verde $\left(87,96{\mathrm{t} . h a^{-1}}^{-1}\right)$ e massa seca $\left(33,1\right.$ t.ha $\left.{ }^{-1}\right)$ quando foram cortadas aos 97 dias após 
Research, Society and Development, v. 10, n. 11, e109101119401, 2021

(CC BY 4.0) | ISSN 2525-3409 | DOI: http://dx.doi.org/10.33448/rsd-v10i11.19401

a semeadura. A opção em adiar 34 dias o corte do sorgo forrageiro proporcionou um aumento de cerca de 1,5 m na altura, 74,5 $\%$ da massa verde e $97,7 \%$ da massa seca das plantas, possivelmente em função das condições climáticas favoráveis (Figura $1)$.

Tabela 1 - Características agronômicas do sorgo forrageiro Agri 002E em função de doses de nitrogênio e épocas do primeiro corte. Terenos - MS. Safra 2018/19.

\begin{tabular}{|c|c|c|c|c|c|c|c|c|c|}
\hline \multirow{2}{*}{$\begin{array}{c}\text { PC } \\
\text { (dias) }\end{array}$} & \multicolumn{4}{|c|}{$\mathrm{N}\left(\mathrm{kg} \cdot \mathrm{ha}^{-1}\right)$} & \multirow[t]{2}{*}{ Média } & \multicolumn{3}{|c|}{ Valor $\mathrm{p}$} & \multirow[b]{2}{*}{$\mathrm{CV}(\%)$} \\
\hline & 0 & 50 & 150 & 200 & & $\mathrm{C}$ & $\mathrm{N}$ & $\mathrm{C}^{*} \mathrm{~N}$ & \\
\hline \multicolumn{10}{|c|}{------------------- Altura de plantas (cm) ------------------ } \\
\hline 63 & 118,65 & 126,98 & 128,48 & 128,61 & $125,68^{\mathrm{A}}$ & $<0,0001$ & 0,9528 & 0,4545 & 3,9 \\
\hline 97 & 291,00 & 265,95 & 276,63 & 272,65 & $276,56^{\mathrm{B}}$ & & & & \\
\hline \multicolumn{10}{|c|}{------------------ Massa verde $\left(\mathrm{kg} \cdot \mathrm{ha}^{-1}\right)$---------------- } \\
\hline 63 & 45.639 & 47.125 & 52.552 & 56.336 & $50.413,00^{\mathrm{A}}$ & $<0,0001$ & 0,2694 & 0,6527 & 19,4 \\
\hline 97 & 77.843 & 88.430 & 99.194 & 86.373 & $87.960,00^{\text {В }}$ & & & & \\
\hline \multicolumn{10}{|c|}{ 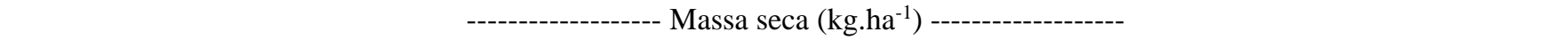 } \\
\hline 63 & 12.129 & 17.767 & 19.394 & 17.707 & $16.749,00^{\mathrm{A}}$ & $<0,0001$ & 0,4061 & 0,7539 & 17,8 \\
\hline 97 & 31.445 & 35.946 & 36.691 & 28.365 & $33.112,00^{\mathrm{B}}$ & & & & \\
\hline \multicolumn{10}{|c|}{ 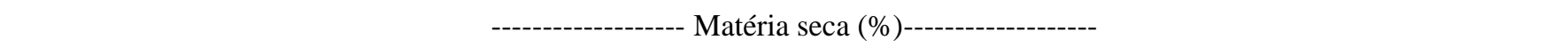 } \\
\hline 63 & 32,18 & 37,30 & 36,19 & 33,06 & 34,80 & 0,8909 & 0,6578 & 0,5743 & 23,3 \\
\hline 97 & 41,38 & 39,94 & 36,68 & 33,22 & 34,68 & & & & \\
\hline
\end{tabular}

Médias seguidas pela mesma letra na coluna não diferem entre si, pelo teste de Tukey a 5\% de probabilidade. PC: primeiro corte. Valor p: probabilidade efeito significativo. $\mathrm{N}$ : doses de nitrogênio, na forma de sulfato de amônio. $\mathrm{C}$ : sorte. $\mathrm{C} * \mathrm{~N}$ : interação entre corte e doses de sulfato de amônio. CV: coeficiente de variação. Fonte: Autores.

\section{Segundo corte}

Verificou-se que as plantas foram mais altas e apresentaram maior produtividade $(\mathrm{P}<0,05)$ quando o SC ocorreu 97 dias após o PC, em relação ao que ocorreu com 63 dias (Tabela 2). Houve interação significativa entre os fatores apenas para massa verde e o desdobramento dos efeitos das interações apontou para um efeito quadrático significativo (p: 0,0379) das doses de $\mathrm{N}$ apenas dentro do SC com 97 dias $\left(\mathrm{y}=0.7801 \mathrm{x}^{2}-103.34 \mathrm{x}+32134 ; \mathrm{R}^{2}\right.$ : 0,9896), indicando aumento de produtividade a partir de $150 \mathrm{~kg} \mathrm{~N} . \mathrm{ha}^{-1}$ (Tabela 6). 
Tabela 2 - Características agronômicas do sorgo forrageiro Agri 002E em função de doses de nitrogênio épocas de segundo corte. Terenos - MS. Safra 2018/19.

\begin{tabular}{|c|c|c|c|c|c|c|c|c|c|}
\hline \multirow{2}{*}{$\begin{array}{l}\text { SC } \\
\text { (dias) }\end{array}$} & \multicolumn{4}{|c|}{$\mathrm{N}\left(\mathrm{kg} \cdot \mathrm{ha}^{-1}\right)$} & \multirow[t]{2}{*}{ Média } & \multicolumn{3}{|c|}{ Valor $\mathrm{p}$} & \multirow[b]{2}{*}{$\mathrm{CV}(\%)$} \\
\hline & 0 & 50 & 150 & 200 & & $\mathrm{C}$ & $\mathrm{N}$ & $\mathrm{C} * \mathrm{~N}$ & \\
\hline \multicolumn{10}{|c|}{----------------- Altura de plantas (cm) ----------------- } \\
\hline 63 & 176,38 & 177,20 & 204,20 & 170,13 & $176,69^{\mathrm{A}}$ & $<0,0001$ & 0,6392 & 0,6678 & 3,26 \\
\hline 97 & 274,05 & 263,10 & 276,25 & 278,73 & $273,03^{\mathrm{B}}$ & & & & \\
\hline \multicolumn{10}{|c|}{ 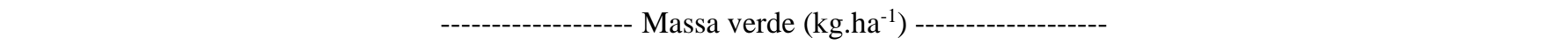 } \\
\hline 63 & $7650^{\mathrm{Ba}}$ & $10688^{\mathrm{Ba}}$ & $7987,5^{\mathrm{Ba}}$ & $8212,5^{\mathrm{Ba}}$ & $8.634,40^{\mathrm{A}}$ & $<0,0001$ & 0,0911 & 0,0222 & 13,26 \\
\hline 97 & $31804^{\mathrm{Ab}}$ & $29576^{\mathrm{Ab}}$ & $33525^{\text {Aab }}$ & $42998^{\mathrm{Aa}}$ & $34.476,00^{\mathrm{B}}$ & & & & \\
\hline \multicolumn{10}{|c|}{ 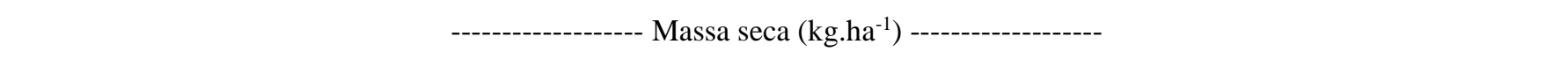 } \\
\hline 63 & 3384,3 & 3932 & 3086,3 & 3219,6 & $3.405,50^{\mathrm{A}}$ & $<0,0001$ & 0,2303 & 0,1312 & 10,61 \\
\hline 97 & 11826,0 & 10146 & 12263 & 20047 & $13.570,00^{\mathrm{B}}$ & & & & \\
\hline \multicolumn{10}{|c|}{ 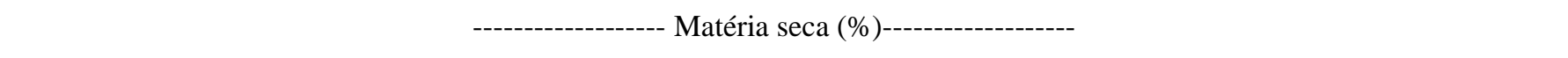 } \\
\hline 63 & 43,26 & 34,87 & 38,69 & 37,69 & 38,54 & 0,8324 & 0,2425 & 0,3135 & 11,15 \\
\hline 97 & 36,55 & 33,53 & 36,30 & 46,02 & 38,10 & & & & \\
\hline
\end{tabular}

Médias seguidas pela mesma letra maiúscula na coluna e minúscula na linha não diferem entre si, pelo teste de Tukey a 5\% de probabilidade. $\mathrm{SC}$ : segundo corte. Valor p: probabilidade efeito significativo. $\mathrm{N}$ : doses de nitrogênio, na forma de sulfato de amônio. C: corte. C*N: interação entre corte e doses de nitrogênio. CV: coeficiente de variação. Fonte: Autores.

Os resultados encontrados concordam com os obtidos por Foloni et al. (2008), que encontraram forte incremento de produtividade de biomassa por meio da adubação com $90 \mathrm{~kg} \mathrm{N.ha}{ }^{-1}$ no rebrote das soqueiras do sorgo forrageiro 'Volumax' cortado a aproximadamente $0,13 \mathrm{~m}$ do solo. Pesquisas desenvolvidas com sorgo sacarino para produção de biocombustível apontaram respostas significativas a doses moderadas de $\mathrm{N}$ que variaram 70 kg.ha-1 (Adams et al., 2015), 90 kg.ha-1 (Sawargaonkar et al., 2013) e 120 kg.ha ${ }^{-1}$ (Tang et al., 2018). Santos et al. (2014) avaliaram o efeito de adubações nitrogenadas sobre a produtividade e a qualidade de fibra de sorgo biomassa para produção de etanol lignocelulósico. Apenas a massa seca total do CMSXS 652 foi afetada pelas doses de N, com produtividade máxima na dose de $130 \mathrm{~kg} \mathrm{ha}^{-1}$.

Evidenciou-se que, independentemente da época do corte, as plantas de sorgo apresentaram maior produtividade quando foram cultivadas por 97 dias (Tabelas 1 e 2), possivelmente por terem um maior período de tempo para expressar seu potencial produtivo.

\section{Comparação entre os cortes}

Comparando-se os dois cortes realizados (PC e SC) após 63 dias de permanência das plantas no campo, notou-se que a altura média das plantas no SC foi cerca de $50 \mathrm{~cm}$ superior em relação àquelas encontradas no PC. Entretanto, percebeu-se uma maior produtividade de massas verde $\left(50,4 \mathrm{t} \mathrm{ha}^{-1}\right)$ e seca $\left(16,8 \mathrm{t}^{-h a^{-1}}\right)$ das plantas no PC (Tabela 3) em relação ao SC. O mesmo foi observado quando o corte ocorreu com 97 dias, onde a produtividade em SC reduziu aproximadamente $60 \%$ daquela observada no PC (Tabela 4).

Estes resultados discordam de Silva et al. (2020), que avaliaram o comportamento do híbrido de sorgo forrageiro Podium em função de três cortes e verificaram que a produtividade de massa verde foi superior nos cortes originados do rebrote em Presidente Prudente, SP. Por outro lado, corroboram com Botelho et al. (2010), que verificaram uma maior 
produtividade de massa verde no primeiro corte $\left(47,4\right.$ t.ha $\left.{ }^{-1}\right)$ em relação à rebrota $\left(32,3\right.$ t.ha $\left.{ }^{-1}\right)$ em quatro cultivares de sorgo forrageiro em Janaúba, MG, pelo fato de ter havido um intenso perfilhamento, aumento na população de plantas por unidade de área e redução da participação de folhas na parte aérea, com plantas mais baixas e menor capacidade de captação de luz pelo dossel.

Tabela 3 - Características agronômicas do sorgo forrageiro Agri 002E, com 63 dias ao campo, em função de doses de nitrogênio e do momento do corte. Terenos - MS. Safra 2018/19.

\begin{tabular}{|c|c|c|c|c|c|c|c|c|c|}
\hline \multirow{2}{*}{$\begin{array}{l}\text { Época do } \\
\text { corte }\end{array}$} & \multicolumn{4}{|c|}{$\mathrm{N}\left(\mathrm{kg} \cdot \mathrm{ha}^{-1}\right)$} & \multirow[t]{2}{*}{ Média } & \multicolumn{3}{|c|}{ Valor $\mathrm{p}$} & \multirow[b]{2}{*}{$\mathrm{CV}(\%)$} \\
\hline & 0 & 50 & 150 & 200 & & $\mathrm{EC}$ & $\mathrm{N}$ & $\mathrm{EC} * \mathrm{~N}$ & \\
\hline \multicolumn{10}{|c|}{---------------- Altura de plantas (cm) ---------------- } \\
\hline $\mathrm{PC}$ & 118,65 & 126,98 & 128,48 & 128,61 & $125,68^{\mathrm{A}}$ & $<0,0001$ & 0,9102 & 0,9719 & 0,57 \\
\hline \multicolumn{10}{|c|}{ 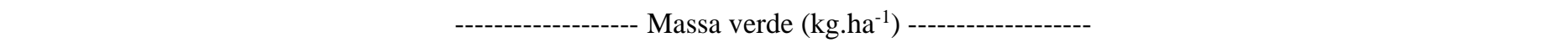 } \\
\hline $\mathrm{PC}$ & 45639 & 47125 & 52552 & 56336 & $50.413,00^{\mathrm{A}}$ & $<0,0001$ & 0,4804 & 0,4801 & 3,05 \\
\hline $\mathrm{SC}$ & 7650 & 10688 & 7987,5 & 8212,5 & $8.634,40^{\mathrm{B}}$ & & & & \\
\hline \multicolumn{10}{|c|}{ 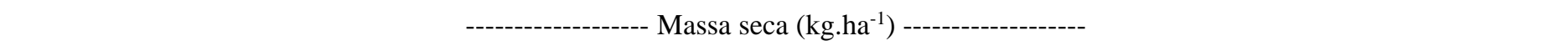 } \\
\hline PC & 43,26 & 34,87 & 38,34 & 37,69 & 34,68 & 0,3318 & 0,9636 & 0,6095 & 24,92 \\
\hline $\mathrm{SC}$ & 32,18 & 37,30 & 36,19 & 33,06 & 38,54 & & & & \\
\hline
\end{tabular}

Médias seguidas pela mesma letra na coluna não diferem entre si, pelo teste de Tukey a 5\% de probabilidade. PC: primeiro corte, em plantas originadas das sementes. SC: segundo corte, em plantas originadas do rebrote de soqueiras. Valor p: probabilidade efeito significativo. N: doses de nitrogênio, na forma de sulfato de amônio. $\mathrm{EC}$ : época do corte. $\mathrm{EC} * \mathrm{~N}$ : interação entre época do corte e doses de nitrogênio. CV: coeficiente de variação. Fonte: Autores.

As plantas apresentaram uma altura média de aproximadamente 2,8 m quando permaneceram 97 dias ao campo, seja antes do PC ou do SC (Tabela 4), comportamento típico de híbridos de sorgo forrageiro considerados "gigantes" (Paziani et al., 2020). Apesar desta variável ser correlacionada com produtividade, deve-se levar em consideração o particionamento de suas estruturas na composição da biomassa. Embora não tenha sido feito a separação das estruturas das plantas neste trabalho, sabese que em sorgos forrageiros altos é encontrada uma maior produção de biomassa atribuída às folhas e colmo, em relação à participação de panículas (Perazzo et al., 2014). Foi observado que as plantas do híbrido Agri 002E apresentaram panículas somente próximo ao SC, independentemente do tempo que estavam sendo cultivadas, porém, eram inférteis e com incidência moderada de doença açucarada ou ergot (Claviceps africana), que atraiu abelhas no final do ciclo da cultura (Grabowski \& Klein, 2017).

A produtividade de massas verde e seca do sorgo forrageiro observada neste trabalho quando as plantas foram cortadas com 97 dias, tanto no PC quanto no SC (Tabelas 1 e 2), esteve superior à apresentada pelas 32 cultivares avaliadas por Perazzo et al. (2014) em Soledade, PA. Da mesma forma, o desempenho do híbrido Agri 002E foi superior aos 24 genótipos cultivados por Lima et al. (2017), que foram colhidos após um único corte após 104 (Goiânia, GO), 117 (Sete Lagoas, MG), 130 (Nova Porteirinha, MG) e 143 dias (Passo Fundo, RS) dias da semeadura e apresentaram variação na produtividade de 
massa verde de 37,15 a 50,3 t.ha-1 ${ }^{-1}$ massa seca de 12,23 a 23,15 3 t.ha ${ }^{-1}$ e altura de 1,72 a 2,83 m. Por meio do cultivo do híbrido Agri 001 para a produção de silagem em Terenos, MS, Rezende et al. (2020) obtiveram a produtividade de 54,25 t.ha-1 de massa verde em um único corte cerca de $0,2 \mathrm{~m}$ acima do nível do solo.

Notou-se que as plantas que receberam o PC com 97 dias produziram aproximadamente 88 t.ha $^{-1}$ de massa verde, enquanto que no SC foi de aproximadamente 34,5 t.ha ${ }^{-1}$, uma produtividade $60,8 \%$ menor e com o mesmo período de permanência do sorgo na área (Tabela 4). Quando as plantas foram adubadas com $200 \mathrm{~kg} \mathrm{N.ha-1}$, não houve diferença (P>0,05) na produtividade de massa seca por hectare entre o PC e o SC. Considerando-se a produtividade de massa seca por hectare, foi observado efeito linear significativo (p: 0,0181) das doses de $\mathrm{N}$ dentro do SC com 97 dias $\left(\mathrm{y}=37.118 \mathrm{x}+9858.7 ; \mathrm{R}^{2}\right.$ : 0,5895) no desdobramento do efeito da interação (Tabela 6).

Tabela 4 - Características agronômicas do sorgo forrageiro Agri 002E, com 97 dias ao campo, em função de doses de nitrogênio e do momento do corte. Terenos - MS. Safra 2018/19.

\begin{tabular}{|c|c|c|c|c|c|c|c|c|c|}
\hline \multirow{2}{*}{$\begin{array}{l}\text { Época do } \\
\text { corte }\end{array}$} & \multicolumn{4}{|c|}{$\mathrm{N}\left(\mathrm{kg} \cdot \mathrm{ha}^{-1}\right)$} & \multirow[t]{2}{*}{ Média } & \multicolumn{3}{|c|}{ Valor $\mathrm{p}$} & \multirow[b]{2}{*}{$\mathrm{CV}(\%)$} \\
\hline & 0 & 50 & 150 & 200 & & EC & $\mathrm{N}$ & $\mathrm{EC} * \mathrm{~N}$ & \\
\hline \multicolumn{10}{|c|}{ - } \\
\hline $\mathrm{PC}$ & 291 & 282,07 & 276,63 & 272,65 & 280,59 & 0,4955 & 0,7319 & 0,6703 & 1,49 \\
\hline $\mathrm{SC}$ & 274,05 & 263,10 & 276,25 & 278,73 & 273,03 & & & & \\
\hline \multicolumn{10}{|c|}{ - } \\
\hline PC & $77843^{\mathrm{Aa}}$ & $88430^{\mathrm{Aa}}$ & 99194 Аа & $86373^{\mathrm{Aa}}$ & $87960^{\mathrm{A}}$ & $<0,0001$ & 0,0339 & 0,0509 & 0,14 \\
\hline $\mathrm{SC}$ & $31804^{\mathrm{Ba}}$ & $29576^{\text {Ba }}$ & $33525^{\mathrm{Bab}}$ & $42998^{\mathrm{Bb}}$ & $34476^{\mathrm{B}}$ & & & & \\
\hline \multicolumn{10}{|c|}{ - Massa seca $\left(\mathrm{kg} \cdot \mathrm{ha}^{-1}\right)$} \\
\hline PC & 31445 Аа & $35946^{\text {Аа }}$ & 36691 Аа & $28365^{\text {Aa }}$ & $33112^{\mathrm{A}}$ & $<0,0001$ & 0,4566 & 0,0417 & 6,01 \\
\hline $\mathrm{SC}$ & $11826^{\mathrm{Bab}}$ & $10146^{\mathrm{Bb}}$ & 12263 Bab & $20047^{\mathrm{Aa}}$ & $13570^{\mathrm{B}}$ & & & & \\
\hline & & & & & & & & & \\
\hline PC & 41,38 & 39,94 & 36,68 & 33,22 & 37,81 & 0,9353 & 0,8848 & 0,1155 & 11,27 \\
\hline $\mathrm{SC}$ & 36,55 & 33,53 & 36,29 & 46,02 & 38,10 & & & & \\
\hline
\end{tabular}

Médias seguidas pela mesma letra maiúscula na coluna e minúscula na linha não diferem entre si, pelo teste de Tukey a 5\% de probabilidade. PC: primeiro corte, em plantas originadas das sementes. SC: segundo corte, em plantas originadas do rebrote de soqueiras. Valor p: probabilidade efeito significativo. N: doses de nitrogênio, na forma de sulfato de amônio. EC: época do corte. EC*N: interação entre época do corte e doses de nitrogênio. CV: coeficiente de variação. Fonte: Autores.

\section{Manejo de cortes}

Ao comparar os manejos dos cortes associados às doses de $\mathrm{N}$ nos 160 dias de permanência do sorgo forrageiro na área experimental, foi observado que estes interagiram somente para produtividade de massa verde (Tabela 5). Somente houve significância a 5\% de probabilidade para o efeito das doses de N quando o PC ocorreu aos 63 dias após a semeadura, sucedido por outro (SC) aos 97 dias após o rebrote das soqueiras. Ajustou-se a produtividade de matéria verde em função das doses $\left(\mathrm{R}^{2}\right.$ : 0,952) a uma equação linear $(y=168.86 x+64093)$.

Analisando-se o efeito dos manejos de corte, obteve-se a maior produtividade $(\mathrm{P}<0,05)$ de matéria verde $\left(96,6\right.$ t.ha $\left.{ }^{-1}\right)$ e seca $\left(37,9\right.$ t.ha $\left.^{-1}\right)$ com o PC aos 97 dias e o SC após 63 dias. Neste manejo as plantas não responderam à adubação nitrogenada em cobertura, provavelmente pela maior produtividade estimada no PC, período que não foram constatados efeitos 
significativos das doses de N (Tabela 1). Nestas condições, aventa-se a hipótese que as plantas do híbrido Agri 002E se desenvolveram adequadamente e empregaram de forma eficiente os $27 \mathrm{~kg}$ N.ha-1 dispensados na semeadura.

O mesmo foi relatado por Marsalis et al. (2010), ao estudarem o comportamento de sorgo forrageiro, híbridos de sorgo com capim sudão e milho recomendados para silagem, submetidos a doses variadas de nitrogênio (106 a $291 \mathrm{~kg}$. ha $\left.{ }^{-1}\right)$ e em diferentes populações de plantas. Após dois anos, concluiu-se que não houve efeito da população de plantas e doses de nitrogênio na produção de matéria seca e em outras propriedades bromatológicas do sorgo forrageiro.

Frias et al. (2018) empregaram cinco doses crescentes de nitrogênio (50, 100, 150, 200 e 250 kg.ha $\left.{ }^{-1}\right)$, parceladas aos 20 e aos 40 dias após a emergência do sorgo forrageiro Volumax na região de Ibiporã, PR e não constataram diferenças nas produções de matéria seca e proteína bruta. Com o objetivo de avaliarem o comportamento de sorgo forrageiro três doses de ureia (80, 120 e $160 \mathrm{~kg} \cdot \mathrm{ha}^{-1}$ ) aplicados de forma parcelada na semeadura e aos 35 dias após a emergência, Maia et al. (2017) verificaram que aos 90 dias o número de folhas vivas, número de perfilho e altura diferiram significativamente, mas o número de folhas mortas, população de plantas, acamamento, plantas quebradas, matéria verde e matéria seca da folha, colmo e panícula não apresentaram diferenças entre os tratamentos. Os resultados deste trabalho apontam para a necessidade de também serem avaliados os efeitos de doses de fertilizantes nitrogenados na composição bromatológica do híbrido Agri 002E, uma vez que esta característica é influenciada pela adubação (Rodrigues Filho et al., 2006).

Tabela 5 - Produtividade total do sorgo forrageiro Agri 002E em função de doses de nitrogênio aplicadas antes do primeiro e segundo cortes e dos manejos de corte. Terenos - MS. Safra 2018/19.

\begin{tabular}{|c|c|c|c|c|c|c|c|c|c|}
\hline \multirow{2}{*}{$\begin{array}{l}\text { Manejos de } \\
\text { corte }\end{array}$} & \multicolumn{4}{|c|}{$\mathrm{N}\left(\mathrm{kg} \cdot \mathrm{ha}^{-1}\right)$ antes do PC e SC } & \multirow[t]{2}{*}{ Média } & \multicolumn{3}{|c|}{ Valor $\mathrm{p}$} & \multirow[b]{2}{*}{$\begin{array}{l}\mathrm{CV} \\
(\%)\end{array}$} \\
\hline & 0 & 50 & 150 & 200 & & M & $\mathrm{N}$ & $\mathrm{M}^{*} \mathrm{~N}$ & \\
\hline \multicolumn{10}{|c|}{ 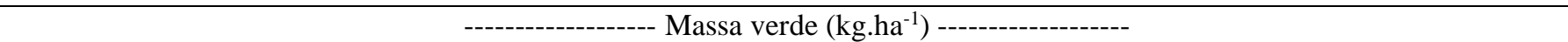 } \\
\hline PC63+SC97 & $61805^{\mathrm{Ab}}$ & 76701 Aab & 86077 Aab & 99333 Aa & $80979^{\mathrm{A}}$ & 0,0103 & 0,0288 & 0,3138 & 1,67 \\
\hline PC97+SC63 & $85493^{\mathrm{Ba}}$ & 99117 Ba & $107181 \mathrm{Aa}$ & $94586^{\mathrm{Aa}}$ & $96594^{\text {B }}$ & & & & \\
\hline \multicolumn{10}{|c|}{ 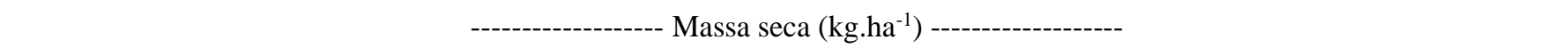 } \\
\hline PC63+SC97 & 23955 & 27912 & 31658 & 37755 & $30320^{\mathrm{A}}$ & 0,0175 & 0,1210 & 0,0760 & 12,97 \\
\hline PC97+SC63 & 34829 & 45365 & 39777 & 31584 & $37889^{\mathrm{B}}$ & & & & \\
\hline \multicolumn{10}{|c|}{ 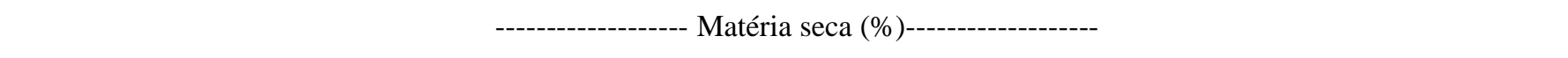 } \\
\hline PC63+SC97 & 34,36 & 35,42 & 38,20 & 39,54 & 36,88 & 0,5585 & 0,9329 & 0,2833 & 16,58 \\
\hline PC97+SC63 & 42,32 & 37,41 & 37,51 & 35,46 & 38,17 & & & & \\
\hline
\end{tabular}

Médias seguidas pela mesma letra na coluna não diferem entre si, pelo teste de Tukey a 5\% de probabilidade. Valor p: probabilidade efeito significativo. N: doses de nitrogênio, na forma de sulfato de amônio. PC63+SC97: primeiro corte aos 63 dias após a semeadura, sucedido por outro aos 97 dias após o rebrote das soqueiras; PC97+SC63: primeiro corte aos 97 dias após a semeadura, sucedido por outro aos 63 dias após o rebrote das soqueiras. $\mathrm{M}^{*} \mathrm{~N}$ : interação entre manejo de cortes e doses de nitrogênio. CV: coeficiente de variação. Fonte: Autores.

Em todas as análises realizadas neste trabalho, percebeu-se que independentemente das doses de $\mathrm{N}$ e a época ou manejo de cortes, não houve diferenças entre os tratamentos $(\mathrm{P}>0,05)$ na matéria seca $(\%)$ das plantas de sorgo (Tabelas 1 a 5$)$, que variou de 34,7 a 38,5\%. Estes valores estão em concordância com os obtidos por Lima et al. (2017), que demostraram uma variação de 37,15 a 50,13 \% no teor de matéria seca de 24 híbridos de sorgo cultivados em diferentes regiões brasileiras.

Há vantagens econômicas ao se realizar o manejo PC63+SC97, pois os incrementos de 15,6 t.ha-1 de massa verde e de 7,7 t.ha ${ }^{-1}$ de massa seca $(\mathrm{P}<0,05)$ podem proporcionar a oferta de silagem por um período de tempo mais prolongado para 
animais de produção (Pereira et al., 2006; Ítavo et al., 2009). Esta maior produção por unidade de área extrapola o cenário da nutrição animal, uma vez que híbridos de sorgo também podem ser empregados em PC ou SC para a produção de cobertura vegetal em sistemas de semeadura direta no Centro-Oeste brasileiro (Pinto et al., 2021).

Além disso, vale ressaltar que a elevada produtividade do híbrido Agri 002E foi superior à relatada para espécies forrageiras muito exigentes em nutrientes (Vilela et al., 2004), como o capim elefante (Jochims \& Lutdke, 2020; Leal et al., 2020), havendo a necessidade de comparar sua composição bromatológica e a viabilidade econômica para diferentes cenários e regiões produtoras. O desenvolvimento das plantas ocorreu em condições de baixa incidência de antracnose (Colletotrichum graminicola), incidência média e prevalente da lagarta do cartucho [Spodoptera frugiperda (JE Smith)] e de pulgão do sorgo [Schizaphis graminum (Rond.)].

Conclui-se que o manejo dos cortes foi a ação mais relevante para se obter aumentos significativos na produtividade de massa seca (Tabela 5). A ausência de aumento na produtividade de massa seca do sorgo forrageiro pela adubação nitrogenada em cobertura indicou que, em função do custo dos fertilizantes (Ogino et al., 2021), o híbrido empregado neste trabalho pode ser considerado sustentável (Marsallis et al., 2010) em regiões com períodos de restrições hídricas recorrentes e limitantes para culturas agrícolas.

Tabela 6 - Desdobramento do efeito da interação entre os fatores doses de nitrogênio (N) e época de corte/manejo em sorgo forrageiro Agri 002E. Terenos - MS. Safra 2018/19.

\begin{tabular}{lccccc}
\hline $\begin{array}{l}\text { Corte ou } \\
\text { Manejo }\end{array}$ & $\begin{array}{c}\text { Idade das plantas } \\
\text { (dias) }\end{array}$ & Variável & Valor $\mathrm{p}$ & $\mathrm{R}^{2}$ & Equação \\
\hline SC & 97 & Massa verde & 0,0379 & 0,999 & $\mathrm{y}=0.7801 \mathrm{x}^{2}-103.34 \mathrm{x}+32134$ \\
SC & 97 & Massa seca & 0,0181 & 0,5895 & $\mathrm{y}=37.118 \mathrm{x}+9858.7$ \\
PC63+SC97 & $63+97$ & Massa verde & 0,0032 & 0,952 & $\mathrm{y}=168.86 \mathrm{x}+64093$ \\
\hline
\end{tabular}

PC: primeiro corte. SC: Segundo corte. PC63+SC97: primeiro corte aos 63 dias após a semeadura, sucedido por outro aos 97 dias após o rebrote das soqueiras. Valor p: probabilidade efeito significativo. Fonte: Autores.

\section{Conclusão}

O sorgo forrageiro Agri 002E apresentou maior produtividade de massa verde e seca a partir de $150 \mathrm{~kg} \mathrm{~N} . \mathrm{ha}^{-1}$ somente quando foi cultivado por 97 dias após o primeiro corte.

Independentemente do período de permanência das plantas no campo, o sorgo forrageiro produziu mais no primeiro corte.

Houve aumento linear de massa verde por hectare com doses crescentes de nitrogênio quando as plantas receberam o primeiro corte aos 63 dias da semeadura, seguido pelo segundo após 97 dias, porém, este não foi o manejo que possibilitou maiores produtividades.

O manejo de cortes considerado mais eficiente por proporcionar as maiores produtividades de matéria verde (96,6 t.ha-1) e seca (37,9 t.ha-1), consistiu no primeiro corte do sorgo forrageiro Agri 002E aos 97 dias, sendo sucedido por outro após 63 dias.

\section{Agradecimentos}

Os autores são gratos ao Conselho Nacional de Desenvolvimento Científico e Tecnológico (CNPq) e à Universidade Federal de Mato Grosso do Sul (UFMS) pela concessão de bolsas de iniciação científica, o suporte financeiro e a 
Research, Society and Development, v. 10, n. 11, e109101119401, 2021 (CC BY 4.0) | ISSN 2525-3409 | DOI: http://dx.doi.org/10.33448/rsd-v10i11.19401

disponibilização dos servidores técnico-administrativos que ajudaram nas operações de campo.

\section{Referências}

Abunyewa, A. A., Ferguson, R. B., Wortmann, C. S. \& Mason, S. C. (2017). Grain sorghum nitrogen use as affected by planting practice and nitrogen rate. Journal of Soil Science and Plant Nutrition, 17(1), 155-166.

Adams, C. B, Erickson, J. E. \& Singh, M. P. (2018). Investigation and synthesis of sweet sorghum crop responses to nitrogen and potassium fertilization. Field Crops Research, 178, 1-7.

Botelho, P. R. F., Pires, D. A. D., Sales, E. C. J., Rocha Júnior, V. R., Jayme, D. G. \& Reis, S. T. (2010). Avaliação de genótipos de sorgo em primeiro corte e rebrota para produção de silagem. Revista Brasileira de Milho e Sorgo, 9(3), 287-297.

Buso, W. H. D, Morgado, H. S., Silva, L. B. \& França, A. F. S. (2011) Utilização do sorgo forrageiro na alimentação animal. PUBVET, 5(23).

Conab. Companhia Nacional de Abastecimento. (2021) Acompanhamento da safra brasileira - grãos, safra 2020/21 - $8^{\circ}$ levantamento, 8(8), 115 p.

Costa N. R., Andreotti, M., Bergamaschine, A. F., Lopes, K. S. M. \& Lima, A. E. S. (2015). Custo da produção de silagens em sistemas de integração lavourapecuária sob plantio direto. Revista Ceres, 62(1), 9-19.

Cptec/Inpe. (2021). Centro de Previsão do Tempo e Estudos Climáticos do Instituto Nacional de Pesquisas Espaciais. https://www.cptec.inpe.br>.

Embrapa. (2013). Sistema brasileiro de classificação de solos. Embrapa.

Fernandes, P. B., Theodoro, G. F., Gurgel, A. L. C., Costa, C. M., Costa, A. B. G., Santana, J. C. S., Silva, M. G. P. \& Bomfim, L. N. (2020). Aspectos relacionados ao potencial forrageiro do sorgo: revisão. PUBVET, 14, 1-7.

Frias, D. B., Coalho, M. R., Costa, M. A. \& Cizanska, I. (2018). Produtividade e qualidade do sorgo forrageiro na região norte do Paraná submetido a diferentes níveis de adubação nitrogenada. Revista Terra \& Cultura: Cadernos de Ensino e Pesquisa, 34, 321 -332.

Gardner, J. C., Maranville, J. W. \& Paparozzi, T. (1994). Nitrogen use efficiency among diverse sorghum cultivars. Crop Science, $34,728-733$.

Grabowski, N. T \& Klein, G. (2017) Microbiology and foodborne pathogens in honey. Critical Reviews in Food Science and Nutrition, 57(9): 1852-1862.

Ítavo, C. C. B. F., Morais, M. G., Ítavo, L. C. V., Souza, A. R. D. L., Davy, F. C. A., Biberg, F. A., Alves, W. B., \& Santos, M. V. (2009). Consumo e digestibilidade de nutrientes de dietas com silagens de grãos úmidos de milho ou sorgo, em ovinos. Arquivo Brasileiro de Medicina Veterinária e Zootecnia, $61(2), 452-459$

Jochims, F. \& Lutdke, A. (2020). Severidade de desfolhação e sua influência no intervalo entre cortes, produtividade e valor nutritivo do capim-elefante BRS Kurumi. Agropecuária Catarinense, 33(2), 42-47.

Kottek, M., Grieser, J., Beck, C., Rudolf, B. \& Rubel, F. (2006). World map of the Köppen-Geiger climate classification updated. Meteorologische Zeitschrift, $15(3), 259-263$

Latinaseeds. (2021). Sorgo gigante boliviano Agri 002E. https://www.latinasementes.com.br/sorgo-agri-002e

Leal, V. N., Machado, R. L., Araujo, L. C., Godoy, M. M., Linhares, A. J. S., Ferreira, J. C. Q., Leopoldino, L. D., Santos, E. A. \& Miyagi, E. S. (2020). Produção e valor nutritivo de forragem de cultivares de capim-elefante em diferentes períodos de rebrotação. Research, Society and Development, 9(11), p.1-9.

Lima, L. O. B., Pires, D. A. A., Moura, M. M. A., Rodrigues, J. A. S., Tolentino, D. C. \& Viana, M. C. M. (2017). Agronomic traits and nutritional value of forage sorghum genotypes. Acta Scientiarum. Animal Sciences. 39(1): 7-12.

Maia, C. P., Barbosa, D. P., Santos, L. F. P., Pereira, N. L. \& Guimarães, A. K. V. (2017). Caracteres produtivos de sorgo híbrido SS-318 submetido a doses de nitrogênio. Agroecossistemas, 9(2), 53-65.

Marsallis, M. A., Angadi, S. V. \& Contreras-Govea, F. E. (2010). Dry matter yield and nutritive value of corn, forage sorghum, and BMR forage sorghum at different plant populations and nitrogen rates. Field Crops Research, 16(1-2), 52-57.

Neumann, M., Restle, J., Souza, A. N. M., Pellegrini, L. G., Zanette, P. M., Nornberg, J. L. \& Sandini, I. E. (2010). Desempenho vegetativo e qualitativo do sorgo forrageiro (Sorghum bicolor X Sorghum sudanense) em manejo de cortes. Revista Brasileira de Milho e Sorgo, 9(3), 298-313.

Ogino, C. M., Costa Junior, G., Popova, N. D. \& Martines Filho, J. G. (2021). Poder de compra, preço e consumo de fertilizantes minerais: uma análise para o centro-oeste brasileiro. Revista de Economia e Sociologia Rural, 59(1), 1-19.

Oliveira, R. P., França, A. F. S., Rodrigues Filho, O., Oliveira, E. R., Rosa, B., Soares, T. V. \& Mello, S. Q. S. (2005). Características agronômicas de cultivares de sorgo (Sorghum bicolor (L.) Moench) sob três doses de nitrogênio. Pesquisa Agropecuária Tropical, 35(1), 45-53.

Paziani, S. F., Freitas, R. S., Duarte, A. P., Ticelli, M., Sawazaki, E. Nussio, L. G., Martins, A. L. M. \& Gallo, P. B. (2020). Sorgos “gigantes” para silagem. Brazilian Journal of Development, 6(12), p. 99936-99946.

Perazzo, A. F., Carvalho, G. G. P., Santos, E. M., Pinho, R. M. A., Campos, F. S., Macedo, C. H. O., Azevêdo, J. A. G. \& Tabosa, J. N. (2014). Agronomic evaluation of 32 sorghum cultivars in the Brazilian semi-arid region. Revista Brasileira de Zootecnia, 43(5), $232-237$. 
Research, Society and Development, v. 10, n. 11, e109101119401, 2021 (CC BY 4.0) | ISSN 2525-3409 | DOI: http://dx.doi.org/10.33448/rsd-v10i11.19401

Perecin, D. \& Cargnelutti Filho, A. (2008). Efeitos por comparações e por experimento em interações de experimentos fatoriais. Ciência e Agrotecnologia, $32(1), 68-72$

Pereira, D. H., Pereira, O. G., Filho, S. C. V., Garcia, R., Oliveira, A. P., Martins, F. H. \& Viana, V. (2006). Consumo, digestibilidade dos nutrientes e desempenho de bovinos de corte recebendo silagem de sorgo (Sorghum bicolor (L.) Moench) e diferentes proporções de concentrado. Revista Brasileira de Zootecnia, 35(1), 282-291.

Pinto, P. H. G., Lima, S. F., Andrade, M. G. O., Contardi, L. M., Ávila, J., Reis, B. O., Bernardo, V. F. \& Vendrusculo, E. P. (2021). Weeds in soybean cultivation with different predecessor cover crops. Revista de Agricultura Neotropical, 8(2), p. 1-10.

Ramadhan, M. \& Muhsin, S. (2021). Evaluation of the response of sorghum to tillage systems and nitrogen fertilization. International Journal of Agronomy. p.1-12.

Restelatto, R., Pavinato, P. S., Sartor, L. R. \& Paixão, S. J. (2013). Production and nutritional value of sorghum and black oat forages under nitrogen fertilization. Grass and Forage Science, 69(4), 693-704.

Rezende, R. P., Golin, H. O., Abreu, V. L. S., Theodoro, G. F., Franco, G. L., Brumatti, R. C., Fernandes, P. B., Bento, A. L. L. \& Rocha, R. F. A. T. (2020). Does intercropping maize with forage sorghum effect biomass yield, silage bromatological quality and economic viability? Research, Society and Development, 9(4), 1-25.

Rodrigues Filho, O., França, A. F. S., Oliveira, R. P., Oliveira, E. R., Rosa, B., Soares, T. V. \& Mello, S. Q. S. (2006). Produção e composição bromatológica de quatro hibrídos de sorgo forrageiro [Sorghum bicolor (1.) Moench] submetidos a três doses de nitrogênio. Ciência Animal Brasileira, 7(1), 37-48.

Santos, F. C., Albuquerque Filho, M. R., Resende, A. V., Oliveira, A. C., Oliveira, M. S. \& Gomes, T. C. (2015). Adubação nitrogenada e potássica na nutrição e na extração de macronutrientes pelo sorgo biomassa. Revista Brasileira de Milho e Sorgo, 14(1), 10-22.

Santos, F. C., Albuquerque Filho, M. R., Resende, A. V., Oliveira, A. C., Gomes, T. C. \& Oliveira, M. S. (2014). Adubações nitrogenada e potássica no sorgo biomassa - produtividade e qualidade de fibra. Revista Brasileira de Milho e Sorgo, 13(1), 1-13.

Sawargaonkar, G. L., Patil, M. D., Wani, S. P., Pavani, E., Reddya, B. V. S. R. \& Marimuthub, S. (2013). Nitrogen response and water use efficiency of sweet sorghum cultivars. Field Crops Research, 149(1), 245-251.

Schneider, J. R., Caron, B. O., Elli, E. F., Schwerz, F. \& Engroff, T. D. (2019). Cutting management modifies the phyllochron, the growth and the production of forage sorghum under the cool environment. Archivos de Zootecnia, 68 (264), 552-560.

Scordia, D., Cosentino, S. L., Mantineo, M., Testa, G. \& Patanè, C. (2021). Nitrogen balance in a sweet sorghum crop in a Mediterranean environment. Agronomy, 11, 1292.

Silva, H. W., Guimarães, C. R. B., Andriguetti, A. C., Godinho, A. M. M., Santos, A. T., Araújo, E. B., Fachiano Júnior, L. C. \& Pereira, C. W. J. (2020). Produtividade de sorgo forrageiro em função do intervalo de corte e da rebrota. Brazilian Journal of Development, 6(3), 14441-14450.

Silva, W. L., Basso, F. C., Ruggieri, A. C., Vieira, B. R., Alves, P. L. C. A. \& Rodrigues, J. A. S. (2012) Características morfogênicas e estruturais de híbridos de sorgo submetidos a adubação nitrogenada. Revista Brasileira de Ciências Agrárias, 7(4), 691-696.

Tang, C., Yang, X., Chen, X., Ameen, A., Xie, G. (2018). Sorghum biomass and quality and soil nitrogen balance response to nitrogen rate on semiarid marginal land. Field Crops Research, 215(1), 12-22.

Theodoro, G. F., Golin, H. O., Silva, M. S., Rezende, R. P. \& Abreu, V. L. S. (2018). Influência de sistemas de preparo na manutenção da palhada e resistência do solo à penetração. Revista de Agricultura Neotropical, 5(2), 25-30.

Tiritan, C. S., Santos, D. H., Minutti, C. R., Foloni, J. S. S. \& Calonego, J. C. (2013). Bromatological composition of sorghum, millet plant and midget guandu at different cut times in intercropping and monoculture. Acta Scientiarum. Agronomy, 35(2), 183-190.

Tolentino, D. C., Rodrigues, J. A. S., Pires, D. A. A., Veriato, F. T., Lima, L. O. B. \& Moura, M. M. A. (2016). The quality of silage of different sorghum genotypes. Acta Scientiarum. Animal Sciences, 38(2), 143-149.

Viana, A. F. P., Cattelam, J., Cattelam, P. M. M., Klein, J. L., Adams S. M., Machado, D. S., Rodrigues, L. S., Brondani, I. L. \& Alves Filho, D. C. (2020). Pastagens de milheto ou sorgo forrageiro para novilhos de corte em fase de crescimento. Research, Society and Development, 9(10), 1-24.

Vilela, L., Soares, W. V., Sousa, D. M. G. \& Macedo, M. C. M. (2004). Calagem e adubação para pastagens. In: Souza, D.M.G. \& Lobato, E. Cerrado: correção do solo e adubação. Brasília: Embrapa, (2a ed.), 416 p.

Zhao, D., Reddy, K. R., Kakani, V. G. \& Reddy, V. R. (2005). Nitrogen deficiency effects on plant growth, leaf photosynthesis, and hyperspectral reflectance properties of sorghum. European Journal of Agronomy, 22, 391-403. 